\title{
BDS satellite clock offset prediction based on a semiparametric adjustment model considering model errors
}

\author{
Xiong Yan $^{1,2^{*}} \mathbb{B}$, Wentao $\mathrm{Li}^{1}$, Yufeng Yang ${ }^{1}$ and Xiong Pan ${ }^{1}$
}

\begin{abstract}
In view of the influence of model errors in conventional BeiDou prediction models for clock offsets, a semiparametric adjustment model for BeiDou Navigation Satellite System (BDS) clock offset prediction that considers model errors is proposed in this paper. First, the model errors of the conventional BeiDou clock offset prediction model are analyzed. Additionally, the relationship among the polynomial model, polynomial model with additional periodic term correction, and its periodic correction terms is explored in detail. Second, considering the model errors, combined with the physical relationship between phase, frequency, frequency drift, and its period in the clock sequence, the conventional clock offset prediction model is improved. Using kernel estimation and comprehensive least squares, the corresponding parameter solutions of the prediction model and the estimation of its model error are derived, and the dynamic error correction of the clock sequence model is realized. Finally, the BDS satellite precision clock data provided by the IGS Center of Wuhan University with a sampling interval of $5 \mathrm{~min}$ are used to compare the proposed prediction method with commonly used methods. Experimental results show that the proposed prediction method can better correct the model errors of BDS satellite clock offsets, and it can effectively overcome the inaccuracies of clock offset correction. The average forecast accuracies of the BeiDou satellites at 6, 12, and $24 \mathrm{~h}$ are 27.13\%, 37.71\%, and $45.08 \%$ higher than those of the conventional BeiDou clock offset forecast models; the average model improvement rates are $16.92 \%, 20.96 \%$, and $28.48 \%$, respectively. In addition, the proposed method enhances the existing BDS satellite prediction method for clock offsets to a certain extent.
\end{abstract}

Keywords: BDS, Satellite clock offset, Model errors, Semiparametric adjustment model, Clock offset forecast

\section{Introduction}

The navigation satellite is a time-based ranging system. The high-precision space-borne atomic clock is the basis of the global navigation satellite system (GNSS). By estimating and forecasting the satellite clock offset parameters of the onboard atomic clock, the time parameters of the precise onboard atomic clock are obtained. The time synchronization of the navigation system and improvement of its related service performance are significant (Guo 2006; Huang et al. 2011; Wang et al. 2017). In the

\footnotetext{
*Correspondence: yx_CUG@163.com

1 School of Information and Engineering, China University of Geosciences, No. 388, Lu Mill Road, Hongshan District, Wuhan, China

Full list of author information is available at the end of the article
}

process of satellite high-speed movement, the spaceborne atomic clock is highly susceptible to the external environment and its own factors, creating difficulty for it to grasp its detailed change laws. Therefore, establishing a precise atomic clock operation model is very difficult, which correspondingly results in inaccurate prediction of satellite clock offsets. In recent years, to improve the accuracy of satellite clock offset predictions, many scholars have proposed a variety of clock offset prediction models and methods, including the polynomial model (Wang et al. 2017; Wang 2017; Huang 2012) (PM), spectrum analysis model (Huang 2012; Zheng et al. 2010) (SA), gray model (Wang 2017; Huang 2012; Zhou et al. 2015) (GM(1,1)), Kalman filter model (Huang et al. 2014; Ai et al. 2016) (KF), time sequence model (Wang 2017; 
Huang 2012; Zhang et al. 2007), least squares support vector machine model (Zhang et al. 2013; Ma et al. 2017) (LSSVM), radial basis neural network model (Xiao et al. 2006) (RBF), wavelet neural network model (Huang et al. 2018; Wang et al. 2016) (WNN), and its related combined forecasting models (Cai et al. 2016), each of which has its own characteristics when performing clock forecasting (Wang 2017; Huang 2012). Among them, the quadratic polynomial model (QP) and the linear model (LP) in the polynomial model (PM) are the most commonly used and representative because of their simple modeling and clear physicalproperties (Wang 2017; Huang 2012). However, because the satellite clock offset is based on multisatellite joint orbit determination, it will be affected by satellite orbital coupling errors (Wang 2017). Moreover, the mechanical modeling of the BeiDou satellite, especially the optical pressure model, has a certain systematic deviation (Zhou et al. 2015). According to the law of error propagation, these will lead to errors in the clock offset model, which will affect the fitting effect and prediction accuracy of the QP and LP models to a certain extent. In addition, the residual of the satellite clock offset contains other colored noises other than white noise, making it difficult for conventional prediction models to fit these nonlinear and non-significant colored noises (Huang et al. 2018). These model errors also have an impact on the accuracy of prediction of satellite clock offsets.

Recently, some scholars have studied the improvement of model errors of the satellite clock offset prediction models and achieved certain results. They mainly use the spectrum analysis method (Wang 2017; Huang 2012; Zheng et al. 2010; Huang et al. 2014; Ai et al. 2016; Zhang et al. 2007, 2013; Ma et al. 2017), and the singular spectrum analysis combined with Fourier band-pass filter method (SSA + FBPF) (Xiao et al. 2006) to obtain the main period of the clock offset sequence so as to correct the periodicity of and improve the fitting and prediction accuracy of the polynomial model. However, some problems arise with correcting the model through periodic item corrections: (1) Because the correct determination of the main period term in the forecast period requires a long and stable clock offset sequence, the correction term of the main period is likely to be unreliable if it is not satisfied; (2) The method of extracting periodic terms will also affect the credibility of the periodic terms extracted from the clock offset sequence, and the effect of model correction is also related to the number of added main periodic correction terms. Sometimes the number of added periodic correction items is inappropriate, which will easily lead to the phenomenon of "over-fitting" of prediction accuracy decline. In addition, fully considering the influence of model errors on prediction modeling of the satellite clock offsets by using only periodic term correction is quite difficult, which to some extent limits the improvement of the prediction accuracy of the satellite clock offset model.

By analyzing and sorting out existing achievements, this paper introduces the semiparametric adjustment model for the clock offset prediction of BeiDou Navigation Satellite System (BDS), and proposes a prediction method for satellite clock offsets based on the semiparametric adjustment model that considers model errors. First, the relationship among the polynomial model, polynomial model with additional periodic term correction, and periodic term correction is analyzed, and the optimum principal periodic term correction is determined. Subsequently, a semiparametric adjustment model is established based on the physical relationship among the phase, frequency, frequency drift, and their periods in the clock offset sequence, which corresponds to the objective and actual changes of the satellite clock offset sequence. The model parameters are estimated using a semiparametric kernel estimation and the corresponding model errors are dynamically corrected. Finally, using the BeiDou satellite clock data available in literature (Ai et al. 2016), the method in this paper is used for experimental processing and is compared with the conventional clock offset prediction method to verify its effectiveness. The results of the example indicate that the proposed satellite clock offset prediction method can compensate for the model errors caused by determining the main period in the forecast and effectively correcting the model errors caused by other factors. Accuracy and forecasting accuracy are significantly improved compared with traditional polynomial models.

\section{Model establishment Basic forecasting model}

The satellite-borne atomic clock currently used by BDS is mainly a cuckoo clock, and its quadratic polynomial model (QP), which includes the phase, frequency, and frequency drift (aging rate), is often used as the BDS satellite clock model (Guo 2006; Huang et al. 2011; Wang et al. 2017). It is given as

$$
y\left(t_{i}\right)=a_{0}+a_{1}\left(t_{i}-t_{0}\right)+a_{2}\left(t_{i}-t_{0}\right)^{2}+\Delta_{i} \quad i=1,2, \ldots
$$

where $y\left(t_{i}\right)$ is the satellite clock offset of the epoch time $t_{i} ; a_{0}, a_{1}$, and $a_{2}$ are the satellite clock parameters, corresponding to the phase, clock speed (frequency), and clock drift (frequency drift), respectively; $t_{0}$ is the satellite clock reference time; $\Delta_{i}$ is the clock offset model error.

\section{Periodic term model}

Because an orbiting satellite is interfered by its orbit and space environment and various perturbations 
(Huang et al. 2018), the clock offset sequence of the solution contains a certain degree of periodic variation law. Therefore, the periodic term model considering the influence of the orbital period and the system's perturbation can be expressed as

$$
\begin{aligned}
y\left(t_{i}\right)= & a_{0}+a_{1}\left(t_{i}-t_{0}\right)+a_{2}\left(t_{i}-t_{0}\right)^{2} \\
& +\sum_{k=1}^{p} A_{k} \sin \left(2 \pi f_{k}+\varphi_{k}\right)+\Delta_{i} \quad i=1,2, \ldots, n
\end{aligned}
$$

where $p$ is the total number of period items; $k$ is the order of the period items attached; $A_{k}, f_{k}$, and $\varphi_{k}$ are the amplitude, frequency, and phase of the corresponding period term.

In the above-mentioned periodic term model, $f_{k}$ and $p$ are mainly determined by spectrum analysis or directly based on the correlation between the significant period of the on-orbit satellite clock and the orbital period (Wang 2017; Sun et al. 2016).

\section{Semiparametric adjustment model considering model errors}

Owing to the satellite clock offset obtained by the multisatellite joint orbit determination, it will be affected by the coupled satellite orbit error (Wang 2017). In addition, the mechanical modeling of the BeiDou satellites, especially the optical pressure model (Zhou et al. 2015, 2016), have a certain systematic deviation, and the quality of the BeiDou satellite clock sequence, as well as other factors (such as colored noise that are not well modeled), will also have a certain degree of influence on the clock offset modeling, which will affect the final clock offset prediction effect. In this regard, based on the error of the periodic term, we classify other relevant systematic errors into non-parametric components $s\left(t_{i}\right)$ and establish a semiparametric adjustment model that considers the model errors, for the corresponding clock offset model to be in accordance with the objective reality. Subsequently, Eq. (2) becomes

$$
\begin{aligned}
y\left(t_{i}\right)= & a_{0}+a_{1}\left(t_{i}-t_{0}\right)+a_{2}\left(t_{i}-t_{0}\right)^{2} \\
& +\sum_{k=1}^{p} A_{k} \sin \left(2 \pi f_{k}+\varphi_{k}\right)+s\left(t_{i}\right)+\Delta_{i}
\end{aligned}
$$

Rewriting Eq. (3) into matrix form, the equation can be expressed as

$$
\left[\begin{array}{c}
y_{1} \\
y 2 \\
\vdots \\
y n
\end{array}\right]=\left[\begin{array}{c}
b_{1} \\
b_{2} \\
\vdots \\
b_{n}
\end{array}\right]\left[\begin{array}{c}
a_{0} \\
a_{1} \\
a_{2} \\
A_{i_{1}} \\
\vdots \\
A_{i_{p}}
\end{array}\right]+\left[\begin{array}{c}
s 1 \\
s 2 \\
\vdots \\
s n
\end{array}\right]+\left[\begin{array}{c}
\Delta 1 \\
\Delta 2 \\
\vdots \\
\Delta n
\end{array}\right]
$$

$$
\begin{aligned}
& \text { where } b_{i} \\
& =\left[\begin{array}{llll}
1\left(t_{i}-t_{0}\right) & \left(t_{i}-t_{0}\right)^{2} \sin \left(2 \pi f_{i_{1}}+\varphi_{i_{1}}\right) & \cdots & \sin \left(2 \pi f_{i_{p}}+\varphi_{i_{p}}\right)
\end{array}\right]
\end{aligned}
$$

$$
\text { Let } L=\left[\begin{array}{c}
y_{1} \\
y 2 \\
\vdots \\
y n
\end{array}\right], \quad B=\left[\begin{array}{c}
b_{1} \\
b_{2} \\
\vdots \\
b_{n}
\end{array}\right], \quad X=\left[\begin{array}{c}
a_{0} \\
a_{1} \\
a_{2} \\
A_{i_{1}} \\
\vdots \\
A_{i_{p}}
\end{array}\right],
$$

$$
S=\left[\begin{array}{c}
s 1 \\
s 2 \\
\vdots \\
s n
\end{array}\right], \quad \Delta=\left[\begin{array}{c}
\Delta 1 \\
\Delta 2 \\
\vdots \\
\Delta n
\end{array}\right], \quad i=1,2, \ldots, n
$$

Subsequently, Eq. (4) can be rewritten into the following vector form:

$$
L=B X+S+\Delta
$$

\section{Model solution}

In this paper, the semiparametric kernel estimation is used to solve the prediction model. First, letting $t_{k}$ be any forecasting moment relative to reference time $t_{0}$, we then define the kernel weight function $W_{i}\left(t_{k}\right)$ for the selected time $t_{k}$ :

$$
\begin{aligned}
W i(t k)= & \frac{K\left((t k-t i) h_{n}^{-1}\right)}{\sum_{j=1}^{n} K\left((t k-t j) h_{n}^{-1}\right)} \\
& i, j=1,2, \ldots, n ; \quad k=1,2, \ldots, l
\end{aligned}
$$

where $K(\bullet)$ is the selected kernel function; $h_{n}$ is the corresponding window width parameter and $h_{n}>0 ; n$ is the number of known clock offset sequences; $l$ is the number of clock offset sequences in the corresponding forecast period.

First, to solve the parameters, assuming that $X$ is known, we can estimate the kernel on the non-parametric component $S$ based on $\left\{t i, L i-b_{i}^{T} X\right\}_{i=1}^{n}$ as follows:

$$
\hat{s}_{k}=\sum_{i=1}^{n} W i(t k)\left(L i-b_{i}^{T} X\right)
$$

From Eq. (9), the residual of the observable $y\left(t_{i}\right)$ is:

$$
v_{k}=b_{k} X+\hat{s}_{k}-L_{k}
$$

If $M_{k}=\left(W_{i}\left(t_{j}\right)\right)_{n \times n}$, the matrix form of the above Eq. (10) is

$$
V=\left(I-M_{k}\right)(B X-L), \quad P
$$

where $I$ is the identity matrix and $P$ the weight matrix, which is set according to the precision of the clock offset 
solution. If no such information is available, it can be set as a unit matrix. According to the least squares criterion.

$$
(B X-L)^{T}\left(I-M_{k}\right)^{T} P\left(I-M_{k}\right)(B X-L)=\min
$$

Subsequently, we can convert the above formula into the following equation:

$$
B^{T}\left(I-M_{k}\right)^{T} P\left(I-M_{k}\right) B X=B^{T}\left(I-M_{k}\right)^{T} P\left(I-M_{k}\right) L
$$

If $\operatorname{rank}(B)=t, t$ is the number of unknown parameters in the corresponding clock model, and that $B$ is the full rank matrix; thus, $B^{T}\left(I-M_{k}\right)^{T} P\left(I-M_{k}\right) B$ is a non-singularity. If $\hat{X}_{k}$ is the estimate of the parameter $X$, the least squares estimate is then

$$
\hat{X}_{k}=\left(B^{T}\left(I-M_{k}\right)^{T} P\left(I-M_{k}\right) B\right)^{-1} B^{T}\left(I-M_{k}\right)^{T} P\left(I-M_{k}\right) L
$$

$\hat{X}_{k}$ is substituted into (9) to obtain an estimate of $\hat{s}_{k}$ :

$$
\hat{s}_{k}=\sum_{i=1}^{n} W i(t k)\left(L i-b_{i}^{T} \hat{X}_{k}\right)=M_{k}\left(L-B \hat{X}_{k}\right)
$$

Therefore, Eqs. (14) and (15) are estimated values of the parametric and non-parametric components of the semiparametric kernel estimation, respectively.

In the solutions of the equations above, initially considering both the selection of the kernel function $K(\bullet)$ and the determination of the corresponding window width $h_{n}\left(h_{n}>0\right)$ is necessary. Several common nuclear function selection schemes are available (Ding 2005; Zhang 2003). The window width parameters of this paper are determined by the generalized cross-validation (GCV) method (Ding 2005).

\section{Selection of kernel function $K(\bullet)$}

$$
\begin{array}{ll}
\text { 1. } & K_{1}(x)=\frac{1}{2} \exp (-|x|) \\
\text { 2. } & K_{2}(x)=\left[\pi\left(1+x^{2}\right)\right]^{-1} \\
\text { 3. } & K_{3}(x)= \begin{cases}\frac{15}{16}\left(1-x^{2}\right)^{2} & (|x| \leq 1) \\
0 & (|x|>1)\end{cases} \\
\text { 4. } & K_{4}(x)= \begin{cases}\frac{1}{\sqrt{2 \pi}}\left[\frac{\sin (x / 2)}{x / 2}\right]^{2} & (x \neq 0) \\
0 & (x=0)\end{cases}
\end{array}
$$

\section{Test and analysis}

\section{Single day forecast example}

To analyze the effect of the clock offset prediction in this paper, BeiDou satellite precision clock products, provided by the IGS Center of Wuhan University, with a sampling interval of $5 \mathrm{~min}$ and Modified Julian Date (MJD) from 57,631.0 to 57,632.0 (2016-8-31 0:00:002016-9-1 0:00:00), are tested (the C13 satellite clock offset sequence is missing during this time period). Additionally, the root mean square (RMS) value is used as the evaluation standard for clock offset modeling and prediction accuracy (Huang 2012; Zheng et al. 2010).

First, because the periodic term model is affected by the number and magnitude (or $T_{k}, f_{k}=1 / T_{k}$ ) of the main periods, the optimum parameters of the main period correction term (i.e., $f_{k}$ and $p$ ) in the semiparametric adjustment model that considers model errors are determined to explore the magnitude of the influence. Because of the limited space of the article and the characteristic of different constellation satellites in the BeiDou satellite navigation system (BDS), three satellites, C01 (GEO), C06 (IGSO), and C12 (MEO), were selected for the test analysis, and the following five schemes were designed:

Scheme 1: The satellite clock offset prediction for $12 \mathrm{~h}$ is conducted using the conventional QP model to the clock offset sequence of the test section.

Scheme 2: The satellite clock offset sequence in the test section is predicted for $12 \mathrm{~h}$ by adding a periodic term model with a correction term of the main period $T_{k}$. The main period added is given as the average orbital period of all types of satellites: the average orbital periods for the GEO, IGSO, and MEO satellites are 23.935, 23.934, and $12.887 \mathrm{~h}$, respectively (Zhou et al. 2015; Wang 2016).

Scheme 3: The satellite clock offset sequence is predicted for $12 \mathrm{~h}$ by adding a period term model with a significant main period $\left(f_{1}\right)$ correction term. The first significant principal period for the GEO, IGSO, and MEO satellites is 12,24 , and $12.911 \mathrm{~h}$, respectively (Zhou et al. 2015; Ai et al. 2016).

Scheme 4: The satellite clock offset prediction is conducted by adding two significant main period ( $f_{1}$ and $f_{2}$ ) correction terms to the clock offset sequence of the test section. The first and second significant principal cycles for the GEO, IGSO, and MEO satellites are 12 and $24 \mathrm{~h} ; 24$ and $12 \mathrm{~h}$; and 12.911 and $6.444 \mathrm{~h}$, respectively (Zhou et al. 2015; Ai et al. 2016).

Scheme 5: The satellite clock offset prediction is conducted by adding three significant main period $\left(f_{1}, f_{2}\right.$, and $f_{3}$ ) correction terms to the clock offset sequence of the test section. The first three significant main periods for the GEO, IGSO, and MEO satellites are 12, 24, and $8 \mathrm{~h} ; 24,12$, and $8 \mathrm{~h}$; and 12.911, 6.444, and 24 h, respectively (Zhou et al. 2015; Ai et al. 2016). 
As can be seen from Table 1, the fitting accuracy of the periodic term model improved compared with the basic forecasting model (QP), and with the increase in the number of corrections added to the main periodic term, the improvement effect was more clear, indicating that the periodic term correction can improve the fitting effect of the basic forecasting model, that is, the model error of QP can be corrected to some extent. From the results of the clock offset prediction, we can see that the addition of the main period term improved the prediction accuracy of the model to a certain extent (except for $\mathrm{C} 12$ ), but the improvement of this accuracy was related to the number of corrections of the main period term. From the results of Schemes 3-5 in Table 1, we can see that for satellite $\mathrm{C} 01$, the prediction effect of the periodic term model was the best when only the first main periodic correction term is added.

For the clock offset for satellite C06, the prediction effect of the periodic term model was the best after adding the first two main periodic correction terms. For satellite $\mathrm{C} 12$, the prediction accuracy of the model became worse when the main period was added. This may be due to the fact that the periodic characteristics of the MEO satellite are not very significant, which led to the introduction of over-fitting model errors into the prediction model when the main period correction term was added, thus reducing the prediction accuracy of the periodic term model. This further indicates that the parameter $p$ of the periodic correction term had a significant influence on the prediction effect of the periodic term model. In addition, we can see that the prediction accuracy of the three types of satellites, especially C06, was significantly reduced by adding three significant principal periods to the prediction model. This is because the quadratic polynomial model adds too many correction terms to the main period term, leading to the introduction of overfitting (model) errors into the prediction model. This also indicates that in the periodic term model, the main period correction terms $p$ to be added should not exceed two, or else the prediction accuracy of the model is easily lowered.

In addition, the results of Schemes 1-3 inTable 1 indicate that QP-T1 $1_{(\text {orb })}$ and QP-T1 had better fitting accuracy than $\mathrm{QP}$, but the fitting accuracy difference between QP-T1 $1_{(\text {orb })}$ and QP-T1 was not significant. The results show that for the period term model that added one main period correction term, whether it was the first significant period of joining each satellite or the average orbit period of the satellite, the improvement effect of the fitting accuracy of the period item model was essentially the same. From the results of QP-T1 $1_{(\text {orb })}$ and QP-T1 in Schemes 2 and 3, we can see that the prediction effect of QP-T1 was generally better than that of QP-T1 $1_{\text {(orb) }}$. This indicates that adding the first significant main period correction term of the satellite to the periodic term model was more advantageous than adding the correction term of one orbit period in the clock offset prediction. Additionally, we can see that the sensitivities of the three satellite periodic term models to the added main period $f_{k}$ were different. The prediction effect adding the first significant main period correction term for satellites C06 and $\mathrm{C} 12$ was similar to that of adding one orbit period correction term. For satellite C01, this difference was more significant, probably because the first significant main period (i.e. $1 / 2$ the average orbital period) of the GEO satellite was more evident than the orbital period error of the satellite.

To consider the influence of periodic errors in BDS without introducing new model errors into corresponding prediction models, this paper makes further studies. According to the experimental results, the parameters of the optimum main period correction terms were $p=1$ and $f_{k}=1 / T_{k}$, respectively (the main periods for the GEO, IGSO, and MEO satellites were 12, 24, and $12.911 \mathrm{~h}$, respectively). On the basis of this periodic term model, the other model errors are also included in the non-parametric components, and the semiparametric adjustment model that considers the model errors

Table 1 Accuracy statistics of satellite clock offset prediction (Unit:ns)

\begin{tabular}{|c|c|c|c|c|c|c|c|}
\hline \multirow[t]{2}{*}{ Scheme category } & \multirow[t]{2}{*}{ Forecast model } & \multicolumn{2}{|l|}{ C01 (GEO) } & \multicolumn{2}{|l|}{ C06 (IGSO) } & \multicolumn{2}{|l|}{ C12 (MEO) } \\
\hline & & Fitting accuracy & Forecast accuracy & Fitting accuracy & Forecast accuracy & Fitting accuracy & $\begin{array}{l}\text { Forecast } \\
\text { accuracy }\end{array}$ \\
\hline Scheme 1 & QP & 0.292 & 6.301 & 1.324 & 37.034 & 0.242 & 1.653 \\
\hline Scheme 2 & QP-T1 (orb) & 0.188 & 10.756 & 0.980 & 14.274 & 0.194 & 1.833 \\
\hline Scheme 3 & QP-T1 & 0.229 & 6.061 & 0.980 & 14.144 & 0.194 & 1.830 \\
\hline Scheme 4 & QP-T2 & 0.186 & 10.104 & 0.792 & 3.493 & 0.170 & 1.732 \\
\hline Scheme 5 & QP-T3 & 0.164 & 13.119 & 0.323 & 38.799 & 0.129 & 6.789 \\
\hline
\end{tabular}

$\mathrm{QP}$ represents the basic prediction model; $\mathrm{QP}-\mathrm{T} 1_{(\mathrm{orb})}$ represents the periodic term model after adding orbit period only; $\mathrm{QP}-\mathrm{Ti}(\mathrm{i}=1,2,3)$ represents the periodic term model after adding the correction of the first i main period term in turn 
of Eq. (3) is established. Short-term and ultra-shortterm predictions for 24,12 , and $6 \mathrm{~h}$ for the clock offset sequence of one-day BDS satellite (because the satellite C13's clock offset sequence is missing, it does not participate in the forecast) are performed, and the results are given in Table 2. Figures 1, 2, 3, 4, 5 and 6 show the fitting and prediction residual sequence of the corresponding model. Among them, the kernel function is selected as $K_{4}(\bullet)$ (According to the experiment, the kernel function $K_{4}(\bullet)$ has better prediction performance and estimation effect on the BDS clock sequence.); the window width parameter $h_{n}$ is determined by $G C V$ methods. Furthermore, for convenience of expression, the semiparametric adjustment model that considers the model errors is represented as Semi-K.

Combined with the corresponding results of Figs. 1, 2 and 3 and Table 2, we can see that the Semi-K model had a better improvement than the QP in both the fitting accuracy and forecasting effect. Among them, the maximum and minimum fitting precisions were 0.284 and $0.043 \mathrm{~ns}$, respectively, which fully proves that Semi-K could fit the BDS clock offset data better. This also illustrates the objective reality that the established model can better characterize the satellite clock difference in dynamic change relative to QP, thus better reflecting the linear characteristics of the $R b$ clock of the BDS system.

In addition, during the test period, satellite $\mathrm{CO6}^{\prime} \mathrm{s}$ data quality was very poor due to the impact of the big clock offset jump between the days in the process of precise orbit determination and calculation of time synchronization clock offset. As can be seen from Figs. 1 and 2, satellite $\mathrm{C} 06$ was more affected by the systematic error factors, and the fitting residual sequence fluctuated significantly.
Figure 3 shows that satellite C06's clock offset sequence was fitted by Semi-K. The residual sequence changes were relatively stable. Additionally, we can see that the accuracy of the clock offset sequence of other satellites was significantly improved, indicating that Semi-K can better separate the model errors in QP and QP-T1, and make the fitted residual sequence more stable.

Figures 4, 5 and 6 show that the prediction accuracy of Semi-K also significantly improved compared with QP and the corresponding QP-T1 model. Additionally, according to the analysis of the results in Table 2, Semi$\mathrm{K}$ effectively corrected the model errors in the prediction stage, especially when the periodic characteristics of the satellite were not very significant; the addition of the periodic term correction would introduce new errors to the model in the prediction stage. On the contrary, Semi$\mathrm{K}$ can effectively compensate for this part of the errors and accordingly achieve good forecasting results.

In addition, among the three types of satellites, the model correction effect of IGSO satellites was the most significant.

The accuracy of QP prediction was very poor when forecasting satellite $\mathrm{C06}$, which is due to the large clock offset jump between the critical points of each day in the process of selecting the orbital arc segment in days during the precise orbit determination and time-synchronization clock offset calculation (Zhu et al. 2008). Table 3 and Fig. 4 clearly show that the accuracy of QP's fitting and prediction was the worst among these satellite clocks, which also indicates that the model errors in the fitting stage had an amplification effect on the prediction phase of the clock offset, which would significantly restrict the prediction accuracy of the model. In addition,

Table 2 Accuracy of clock offset prediction for Semi-K (Unit:ns)

\begin{tabular}{|c|c|c|c|c|c|c|c|c|c|c|c|c|}
\hline \multirow[t]{2}{*}{ PRN } & \multicolumn{3}{|c|}{ Fitting accuracy } & \multicolumn{3}{|c|}{ Forecast accuracy/24 h } & \multicolumn{3}{|c|}{ Forecast accuracy/12 h } & \multicolumn{3}{|c|}{ Forecast accuracy/6 h } \\
\hline & QP & QP-T1 & Semi-K & $\mathrm{QP}$ & QP-T1 & Semi-K & $\mathrm{QP}$ & QP-T1 & Semi-K & QP & QP-T1 & Semi-K \\
\hline C01 & 0.292 & 0.229 & 0.055 & 16.564 & 16.018 & 15.071 & 6.301 & 6.061 & 5.654 & 2.001 & 1.837 & 1.595 \\
\hline $\mathrm{CO} 2$ & 0.380 & 0.192 & 0.092 & 6.271 & 5.849 & 3.887 & 5.210 & 4.008 & 3.766 & 4.055 & 1.234 & 3.137 \\
\hline C03 & 0.390 & 0.132 & 0.043 & 4.169 & 4.809 & 0.470 & 3.138 & 2.449 & 0.439 & 2.696 & 1.658 & 0.447 \\
\hline CO4 & 0.750 & 0.261 & 0.241 & 13.879 & 10.674 & 9.836 & 6.353 & 6.571 & 6.350 & 3.088 & 4.719 & 4.688 \\
\hline C05 & 0.570 & 0.346 & 0.105 & 12.169 & 6.414 & 0.769 & 6.694 & 3.457 & 0.219 & 4.150 & 1.328 & 0.220 \\
\hline Co6 & 1.324 & 0.980 & 0.089 & 77.767 & 31.410 & 7.444 & 37.034 & 14.144 & 1.412 & 18.952 & 8.599 & 1.830 \\
\hline CO7 & 0.600 & 0.193 & 0.078 & 14.099 & 2.555 & 0.597 & 5.497 & 1.831 & 0.346 & 1.731 & 1.188 & 0.467 \\
\hline C08 & 0.390 & 0.323 & 0.246 & 16.025 & 5.312 & 2.796 & 6.707 & 1.296 & 1.151 & 4.429 & 1.642 & 1.550 \\
\hline CO9 & 0.180 & 0.148 & 0.046 & 8.735 & 7.277 & 5.421 & 4.740 & 3.737 & 2.981 & 2.587 & 1.889 & 1.659 \\
\hline C10 & 0.670 & 0.249 & 0.248 & 8.271 & 2.949 & 2.940 & 2.120 & 0.802 & 0.790 & 0.467 & 0.766 & 0.767 \\
\hline C11 & 0.310 & 0.284 & 0.280 & 0.689 & 0.521 & 0.503 & 0.634 & 0.667 & 0.573 & 0.825 & 0.872 & 0.736 \\
\hline $\mathrm{C} 12$ & 0.242 & 0.278 & 0.055 & 1.543 & 0.690 & 5.418 & 1.653 & 0.544 & 0.415 & 1.639 & 0.675 & 0.363 \\
\hline C14 & 0.160 & 0.148 & 0.051 & 2.156 & 2.165 & 0.517 & 1.206 & 1.227 & 0.386 & 0.720 & 0.831 & 0.350 \\
\hline
\end{tabular}

QP represents the basic prediction model; QP-T1 represents the periodic term model after adding the correction of the first main period term; Semi-K represents the semiparametric adjustment model considering model errors 


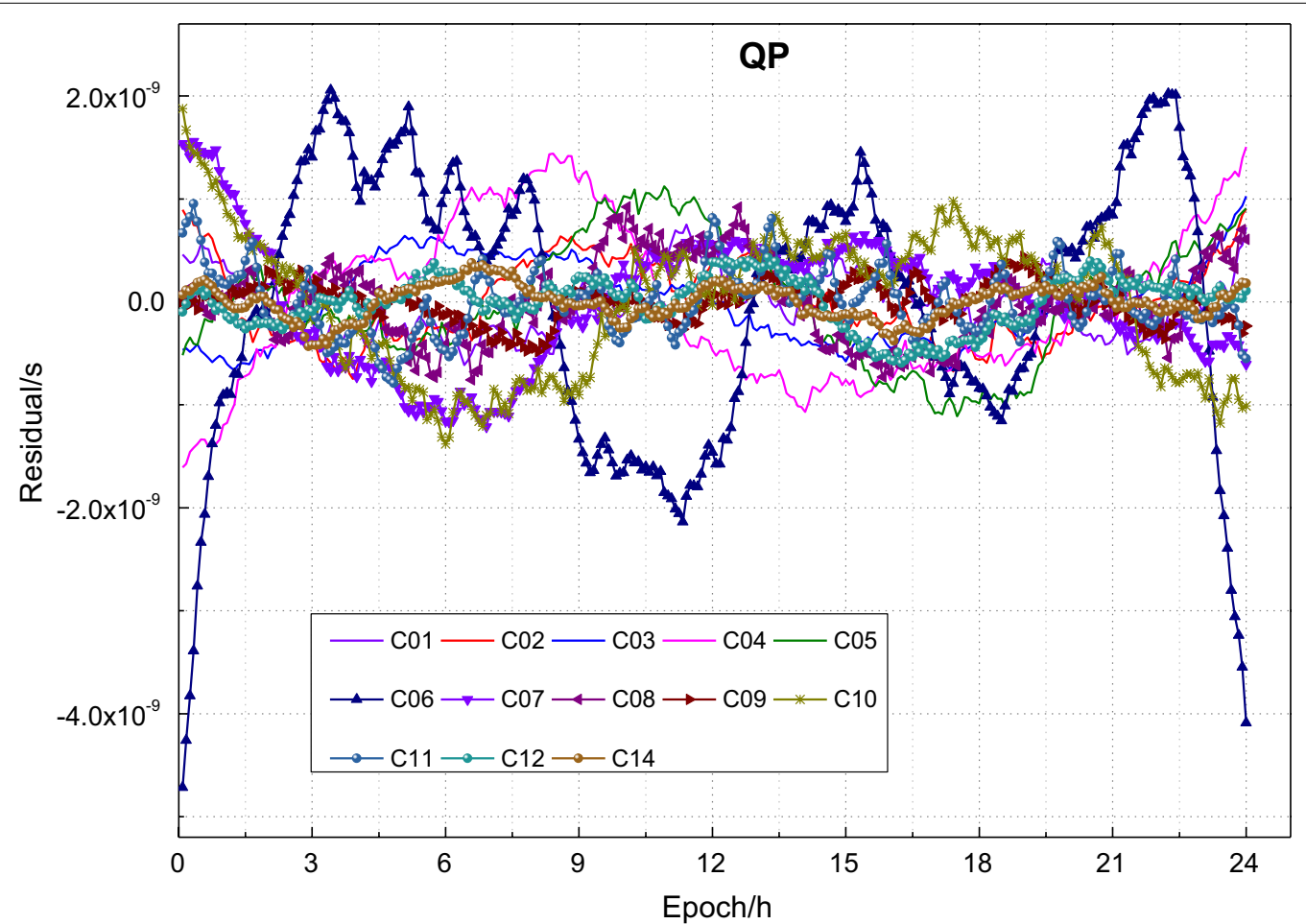

Fig. 1 Fitting residual sequence of QP model

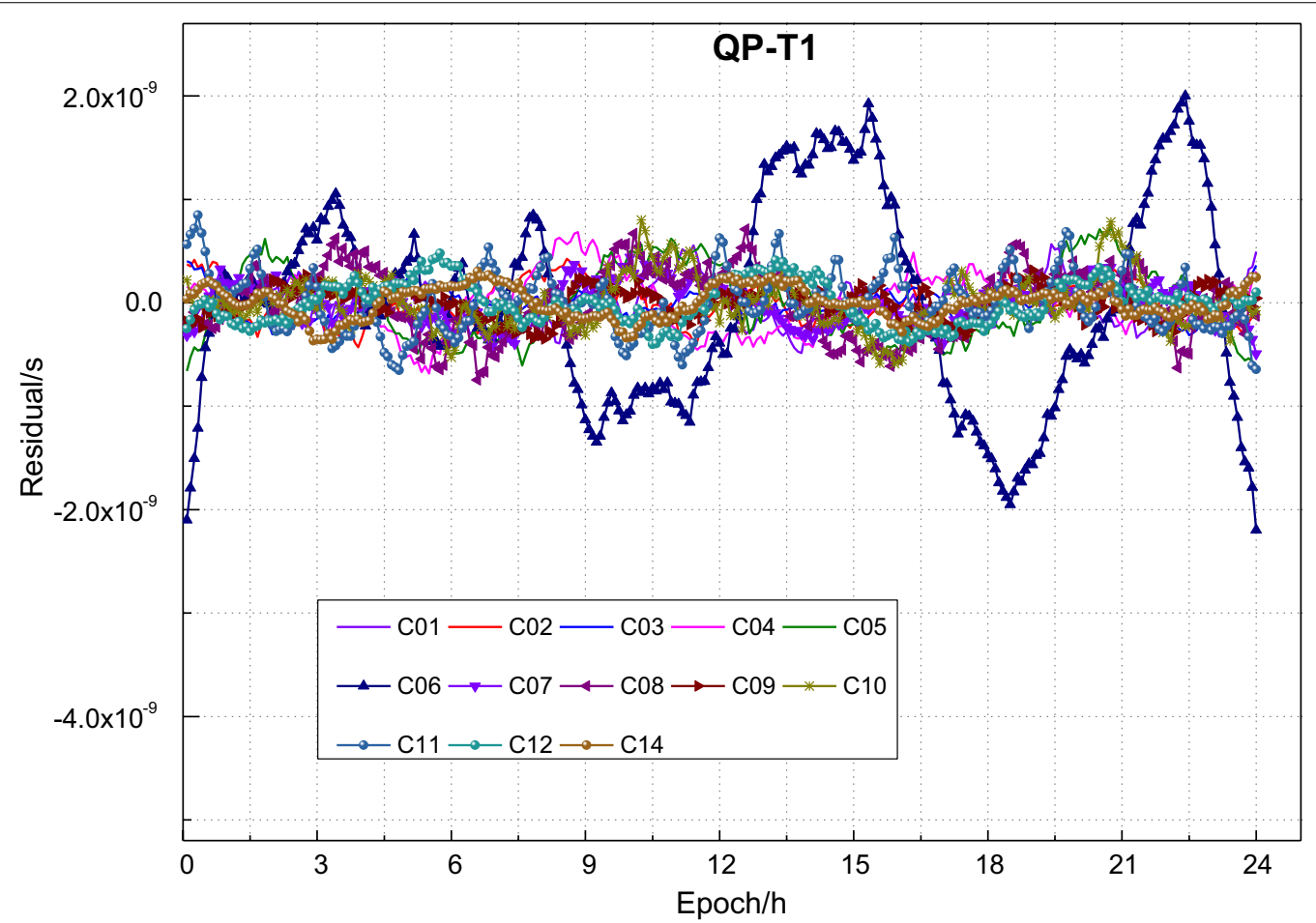

Fig. 2 Fitting residual sequence of QP-T1 model 


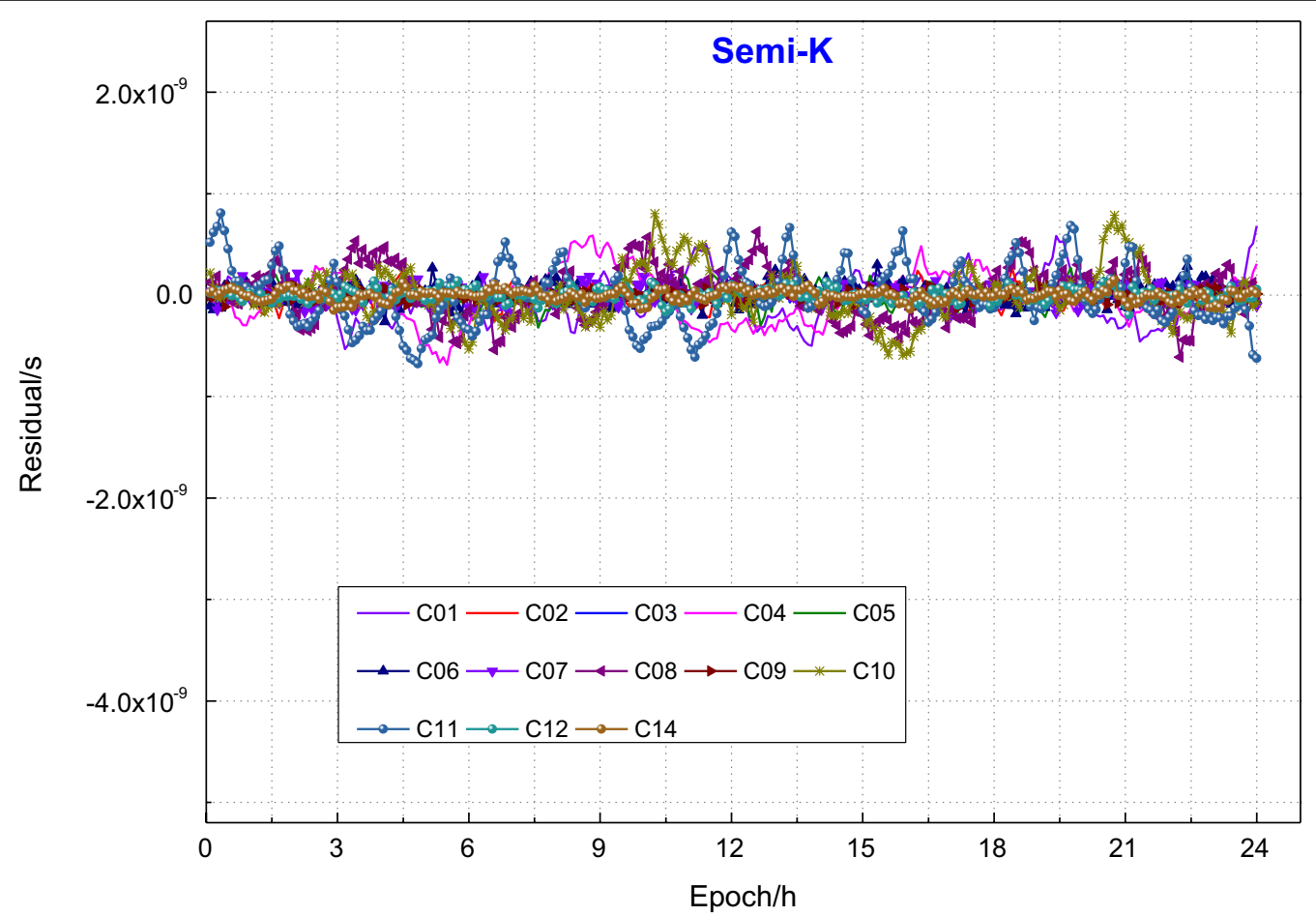

Fig. 3 Fitting residual sequence of Semi-K model

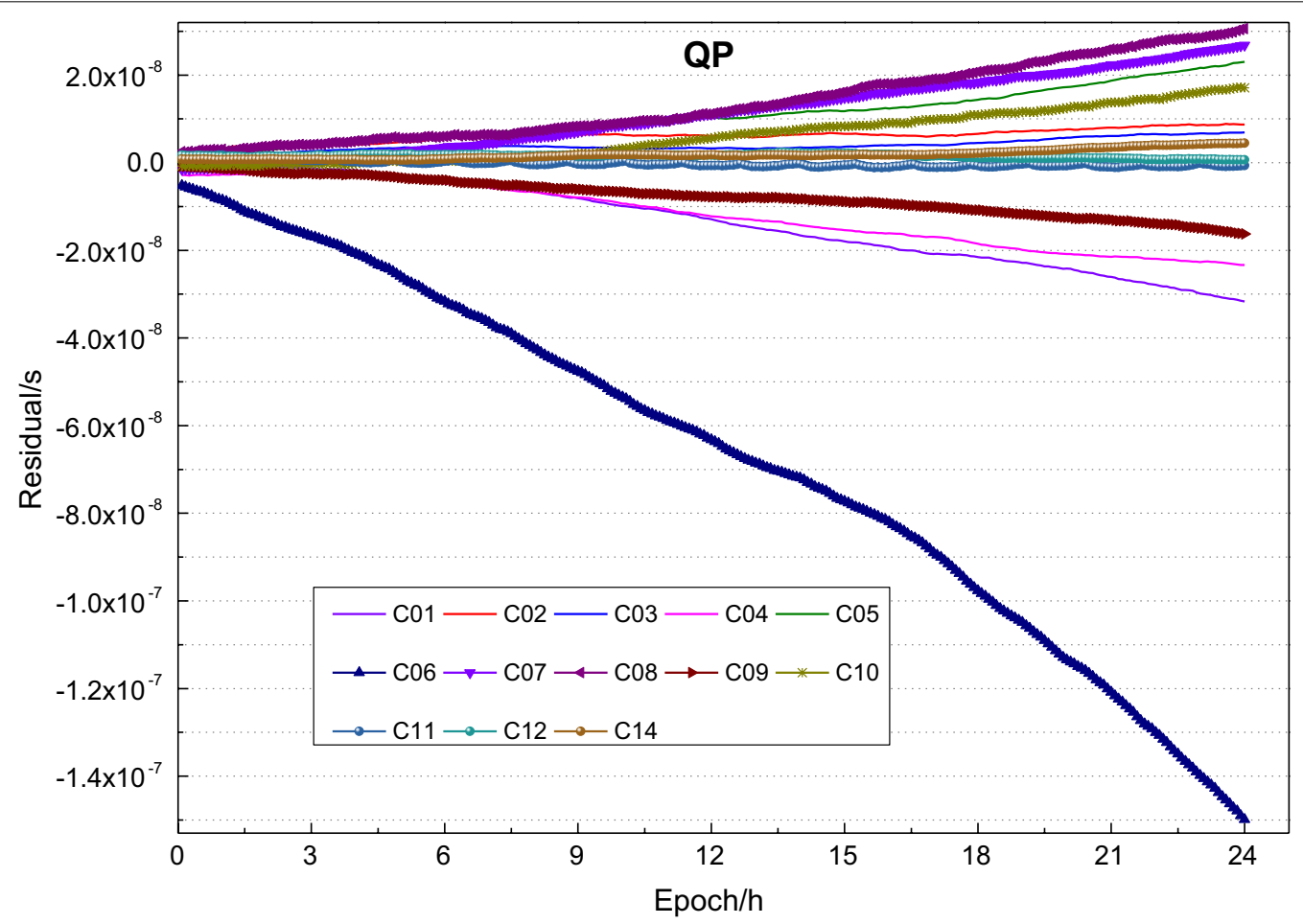

Fig. $424 \mathrm{~h}$ prediction residual sequence of QP model 


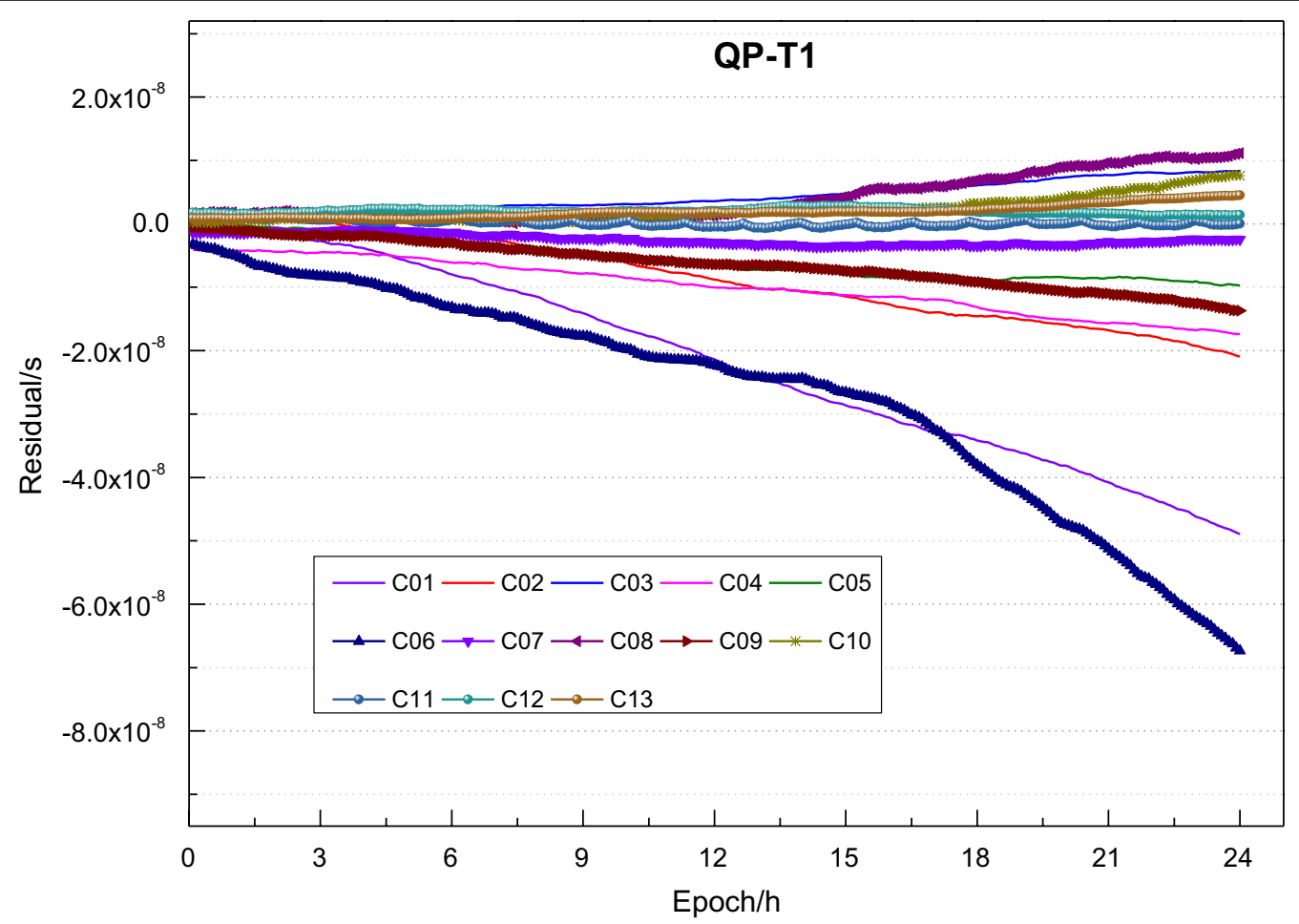

Fig. $524 \mathrm{~h}$ prediction residual sequence of QP-T1 model

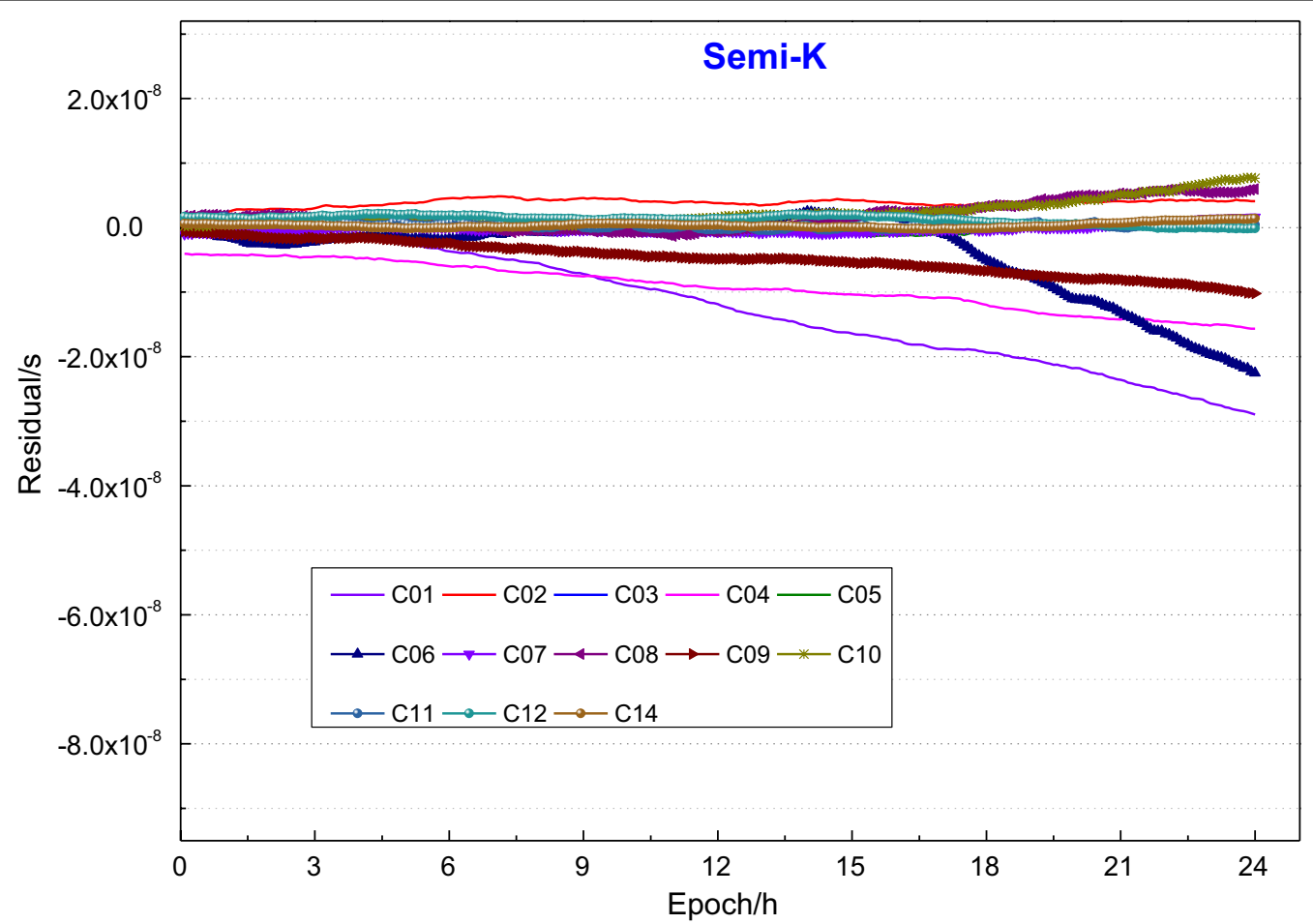

Fig. $624 \mathrm{~h}$ prediction residual sequence of Semi-K model 
this proves to a certain extent that Semi-K can effectively eliminate the systematic errors in the BDS clock offset sequence, and improve the solution accuracy of the model and obtain a better prediction effect.

\section{Multi-day forecast example}

The forecast analysis was conducted using single-day clock offset products, and the amount of data was relatively small, to more accurately reflect the correction effect and prediction accuracy of the model. In this study, the forecasting test was conducted using the corresponding clock products of MJD 57,624.0-57,641.0 (2016-8-24 0:00:00 2016-9-10 0:00:00) available in $\mathrm{Ai}$ et al. (2016) for 18 days. Among them, because the clock offset sequence of MJD 57,635.0-57,636.0 has phase modulation, it did not participate in the relevant forecast, and the forecast results are shown in Figs. 7 and 8 and Table 3. Moreover, to compare the quantitative relationship between the prediction accuracy of the model before and after the improvement, the model improvement rate of Zhou et al. (2015) was introduced in this study. The corresponding results are shown in Table 4 .

$$
\rho=\frac{\left(R M S_{\text {old }}-R M S_{\text {new }}\right)}{R M S_{\text {old }}} \times 100 \%
$$

where $R M S_{\text {old }}$ and $R M S_{\text {new }}$ are the RMS values of the improved front and rear models, respectively.

As can be seen from Fig. 7, the periodic term and Semi-K models significantly improved the average fitting accuracy of QP model in two weeks, which indicates that the periodic error of the clock offset sequence and other unmodeled errors had a significant impact on the fitting effect of the QP model. Among the models, Semi$K$ exhibited the most significant fitting effect. For satellite C06 with poor clock offset data quality, the fitting accuracy improved by at least one order of magnitude, which fully demonstrates that the Semi-K model has a good correction effect on model errors, and is more consistent with the variation characteristics of clock offset sequence. According to the prediction accuracy analysis in Fig. 8 and Table 3, the prediction accuracy of the three models decreased with the increase in the prediction duration, but the accuracy of the periodic term and Semi-K models declined more gradually than that of QP. In addition, Table 3 shows that the average prediction accuracy of the Semi-K model was 27.1037.71, and 45.08\% higher than that of the conventional QP model in the clock offset prediction of the BeiDou satellite at 6,12 , and $24 \mathrm{~h}$, respectively. Among the three satellites, MEO satellites had the best prediction accuracy and the best prediction performance, indicating that the quality of the clock offset data of MEO satellites is better, and their periodic characteristics and other model errors are not significant. For GEO and IGSO satellites, the prediction accuracy of the periodic term and Semi-K models improved to a higher degree than that of the MEO model, indicating that considering the correction of model errors caused by the periodic term and other factors in the clock offset prediction is necessary.

Finally, to quantitatively analyze the model improvement of the Semi-K model, Table 4 shows the result statistics of its improvement rates.

We can see from Table 4 that, with the increase in the forecast duration, the improvement effect of Semi-K on the accuracy of various satellite clock offset predictions

Table 3 Prediction of clock offset in two weeks of Semi-K model (Unit:ns)

\begin{tabular}{|c|c|c|c|c|c|c|c|c|c|c|}
\hline \multirow[t]{2}{*}{ Satellite type } & \multirow[t]{2}{*}{ PRN } & \multicolumn{3}{|c|}{ Forecast accuracy/24 h } & \multicolumn{3}{|c|}{ Forecast accuracy/12 h } & \multicolumn{3}{|c|}{ Forecast accuracy/6 h } \\
\hline & & QP & QP-T1 & Semi-K & QP & QP-T1 & Semi-K & $\mathrm{QP}$ & QP-T1 & Semi-K \\
\hline \multirow[t]{5}{*}{ GEO } & C01 & 12.15 & 11.79 & 11.07 & 7.34 & 7.42 & 7.21 & 5.36 & 5.50 & 5.07 \\
\hline & $\mathrm{CO} 2$ & 7.16 & 6.51 & 4.73 & 4.99 & 4.24 & 3.72 & 3.83 & 3.63 & 3.20 \\
\hline & C03 & 2.60 & 2.45 & 2.08 & 1.91 & 1.79 & 1.65 & 1.66 & 1.59 & 1.14 \\
\hline & C04 & 13.53 & 10.38 & 8.34 & 8.54 & 7.30 & 6.24 & 6.38 & 5.99 & 5.49 \\
\hline & C05 & 13.62 & 13.05 & 7.06 & 7.83 & 8.76 & 5.20 & 4.86 & 6.36 & 4.46 \\
\hline \multirow[t]{5}{*}{ IGSO } & C06 & 61.59 & 25.21 & 12.36 & 33.23 & 14.22 & 7.69 & 18.50 & 9.18 & 5.67 \\
\hline & C07 & 11.79 & 10.45 & 7.46 & 7.07 & 6.49 & 5.21 & 5.49 & 5.05 & 4.50 \\
\hline & C08 & 6.48 & 6.41 & 4.66 & 4.25 & 4.06 & 3.89 & 3.71 & 3.53 & 3.43 \\
\hline & C09 & 10.97 & 7.33 & 6.14 & 7.86 & 5.38 & 4.98 & 6.24 & 4.82 & 4.65 \\
\hline & C10 & 22.51 & 22.40 & 20.86 & 10.01 & 9.75 & 8.31 & 5.78 & 5.04 & 4.79 \\
\hline \multirow[t]{3}{*}{ MEO } & C11 & 5.31 & 5.23 & 4.50 & 4.77 & 4.76 & 4.37 & 4.67 & 4.63 & 4.30 \\
\hline & C12 & 4.55 & 4.50 & 3.83 & 3.90 & 3.88 & 3.50 & 3.76 & 3.70 & 3.46 \\
\hline & C14 & 5.57 & 5.45 & 4.57 & 4.98 & 4.87 & 4.48 & 4.85 & 4.70 & 4.56 \\
\hline
\end{tabular}




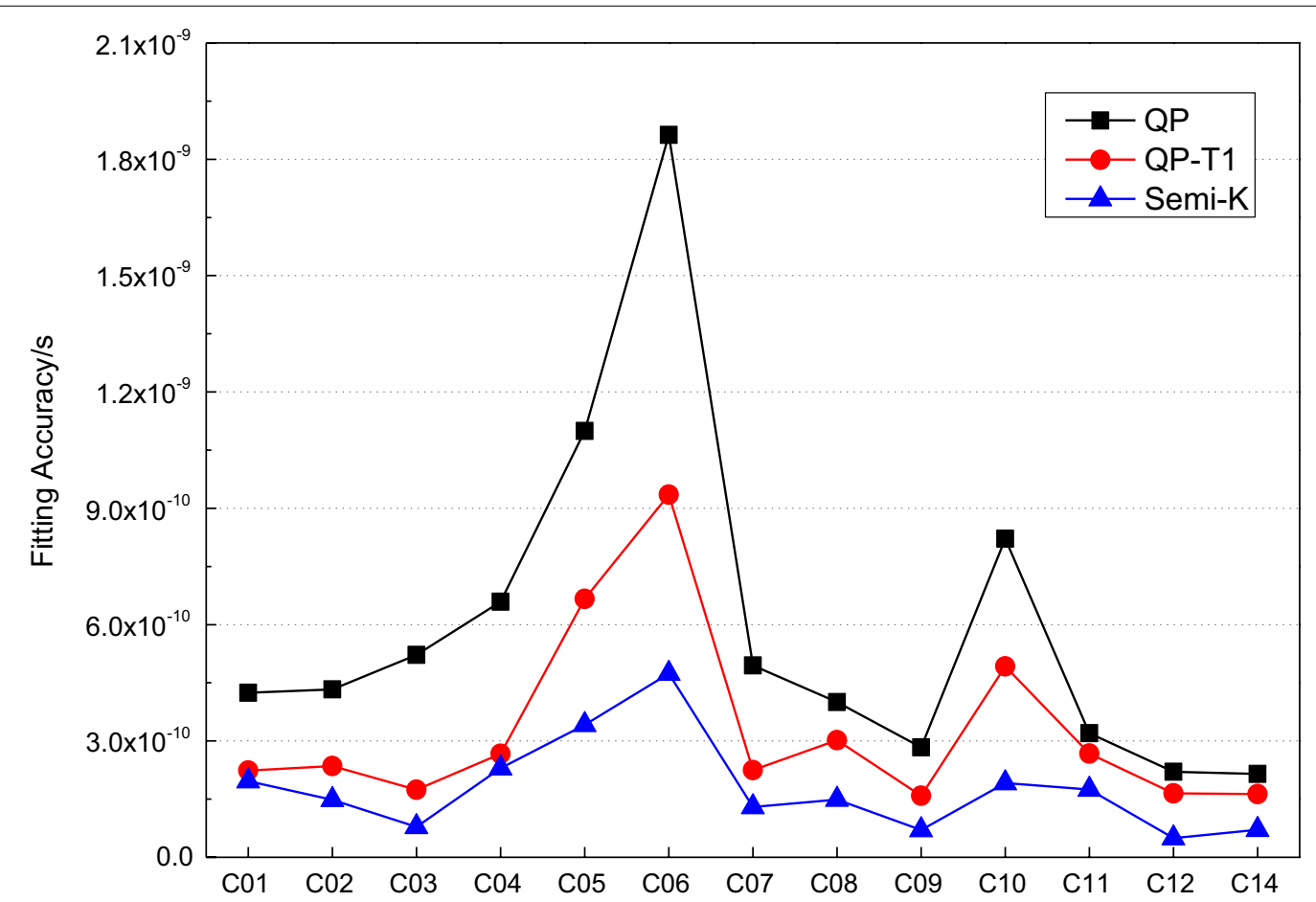

Fig. 7 Statistical fitting accuracy of Semi-K model

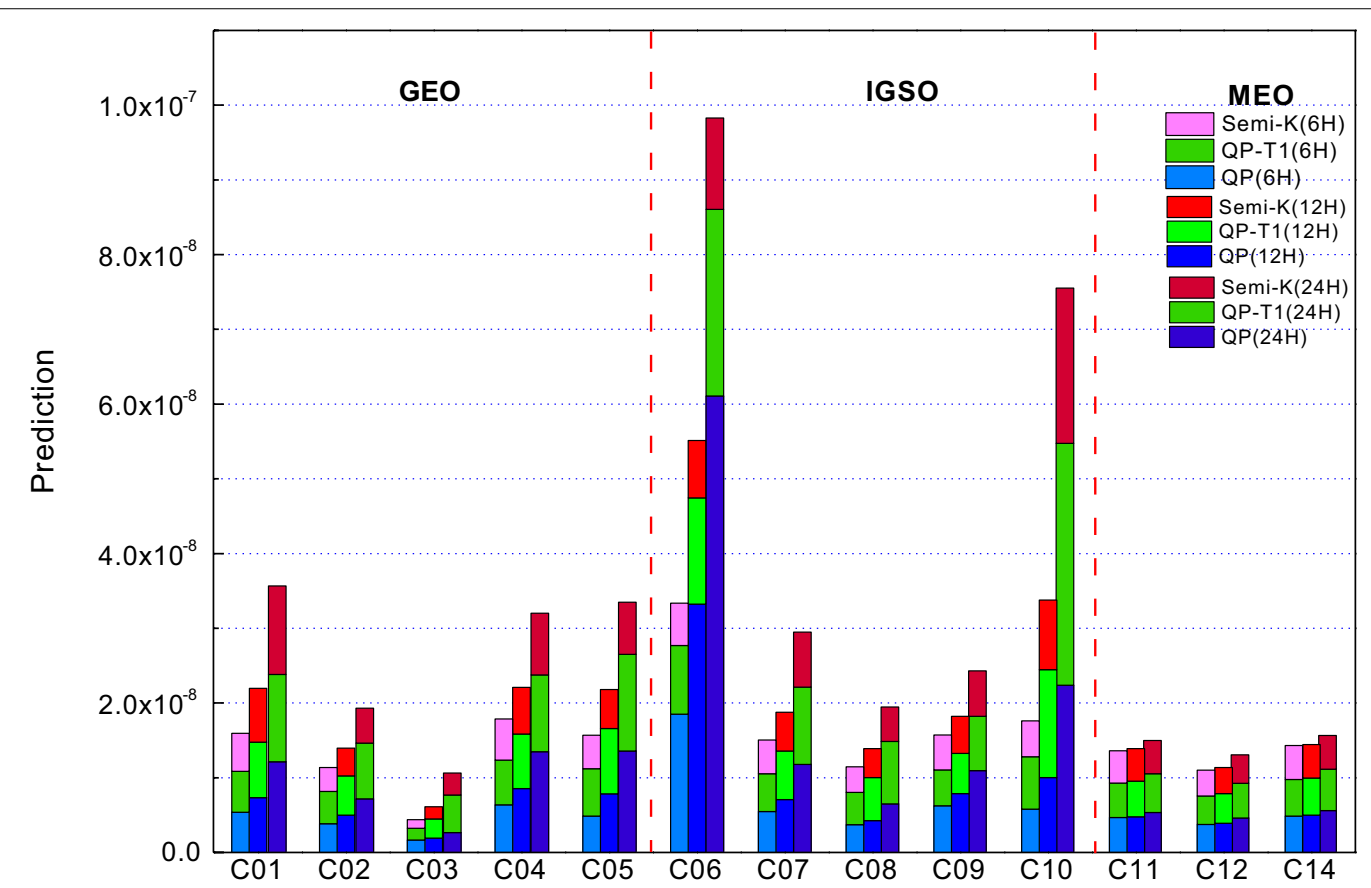

Fig. 8 Cylindrical stacking map predicted by Semi-K model 
Table 4 Model improvement rate of Semi-K model relative to QP model

\begin{tabular}{llcl}
\hline Satellite type & \multicolumn{3}{l}{ Forecast duration } \\
\cline { 2 - 4 } & $\mathbf{6} \mathbf{h}(\mathbf{\%})$ & $\mathbf{1 2} \mathbf{h}(\%)$ & $\mathbf{2 4} \mathbf{h}(\%)$ \\
\hline GEO & 15.07 & 20.27 & 29.87 \\
IGSO & 27.51 & 33.05 & 39.22 \\
MEO & 7.29 & 9.56 & 16.34 \\
\hline
\end{tabular}

was more significant. The improvement rate of the average model of 6,12 , and $24 \mathrm{~h}$ was $16.92,20.96$, and $28.48 \%$ higher than that of the conventional QP model, respectively. The accuracy of MEO satellites with better clock quality improved by approximately $11 \%$. The accuracy of prediction of clock offset for the IGSO satellites improved most significantly, with the accuracy increased by approximately $33 \%$, indicating that IGSO satellites are significantly affected by model errors. Therefore, we recommend optimizing the corresponding BeiDou satellite precise orbit determination model and solution scheme to improve the accuracy of IGSO satellite orbit and clock products.

\section{Conclusions}

At present, the conventional BeiDou clock offset prediction model is affected by model errors. In this paper, a semiparametric adjustment model for BDS satellite clock offset prediction that considers model errors is proposed and constructed, and the corresponding algorithm is designed and implemented. The correction effect and prediction effect of the model are verified by comparing the BDS clock data for two weeks, and the following conclusions are drawn:

(1) To some extent, the model errors of the satellite clock offset can be corrected by the periodic term correction to improve the model fitting and prediction accuracy. However, the effect is unstable, which is significantly affected by the precision of and number of correction items for the periodic term.

(2) For the periodic term model, the parameter $p$ of its main period correction term should not be greater than 2, or else an over-fitting (model) error is easily introduced to the model. For IGSO and MEO satellites, adding a periodic term model with the main period of the satellite's average orbital period and a periodic term model with one main period being the first significant main period $f_{1}$ are essentially consistent in the fitting and forecasting effects; for GEO satellites, the period error of the first signifi- cant main period ( $1 / 2$ average orbit period) is more significant than the orbital period error of the satellite. The effect of both cases, a period term model with a main period as the average orbital period of the satellite and the period term model with the main period as the first significant main period, is essentially consistent in the fitting accuracy, but the former has a better effect in forecast accuracy.

(3) The Semi-K prediction method in this paper can better describe the linear characteristics of the BDS system's $R b$ clock to better correct the model errors contained in the BDS clock offset. With an increase in the prediction time, the model improvement rate increases, with the average model improvement rates for 6,12 , and $24 \mathrm{~h}$ being $16.92,20.96$, and $28.48 \%$, respectively.

(4) According to the analysis of short-term and ultrashort-term clock offset prediction results in two weeks, the average prediction accuracies of BeiDou satellites at 6, 12, and $24 \mathrm{~h}$ are 27.13, 37.71, and $45.08 \%$ higher than that of the conventional QP model, respectively.

In addition, the BDS satellite clock offset prediction method, which accounts for the model errors, has certain universality for GPS, Galileo, and GLONASS systems.

\section{Acknowledgements \\ Thanks to the product support provided by IGS data center of Wuhan University.}

\section{Authors' contributions}

$\mathrm{XY}$ proposed the idea of applying the semiparametric adjustment model to BDS satellite clock offset prediction and derived the corresponding formula, compiled the relevant procedures, and was a major contributor in writing the manuscript. WL collected and compiled the data of the clock offset prediction. YY drew the relevant charts and helped write the manuscript. XP further refined the sentences of the manuscript and gave many constructive suggestions. All authors read and approved the final manuscript.

\section{Funding}

Funding was provided by China University of Geosciences, Wuhan (CN) (Grant No. 41374017).

\section{Availability of data and materials}

The datasets generated and/or analyzed during the current study are available in Ai et al. (2016). https://doi.org/10.11947/j.agcs.2016.f034 (the detailed link address: http://www.igs.gnsswhu.cn/index.php/Home/DataProduct/).

\section{Competing interests}

The authors declare that they have no competing interests.

\section{Author details}

1 School of Information and Engineering, China University of Geosciences, No. 388, Lu Mill Road, Hongshan District, Wuhan, China. ${ }^{2}$ Chihiro Location Network Co., Ltd., Bay Valley Science and Technology Park, No. 38, Lane 1688, Guoquan North Road, Yangpu District, Shanghai, China.

Received: 19 June 2019 Accepted: 24 December 2019

Published online: 28 September 2020 


\section{References}

Ai, Q. S., Xu, T. H., Sun, D. W., et al. (2016). The prediction of BeiDou satellite clock bias based on periodic term and starting point deviation correction. Acta Geodaetica et Cartographica Sinica, 45(S2), 132-138. https://doi. org/10.11947/j.AGCS.2016.F034.

Cai, C. L., He, C. W., \&Wei, Z. C. (2016). A high-precision correction method of ultra-rapid ephemeris clock bias prediction for GPS block IIR-M satellite. Acta Geodaetica et Cartographica Sinica, 45(07), 782-788. https://doi. org/10.11947/j.AGCS.2016.20160017.

Ding, S. J. (2005). Survey data modeling and semiparametric estmating. Wuhan: Wuhan University.

Guo, H. R. (2006). Study on the analysis theories and algorithms of the time and frequeney charaeterization for atomie clocks of navigation satellites. Zhengzhou: PLA Information Engineering University.

Huang, G. W. (2012). Research on algorithms ofof precise clock offset and quality evaluation of GNSS satellite clock. Xi'an: Chang'an University.

Huang, G. W., Yang, Y. X., \& Zhang, Q. (2011). Estimate and predict satellite clock error using adaptively robust sequential adjustment with classified adaptive factors based on opening windows. Acta Geodaetica et Cartographica Sinica, 40(01), 15-21.

Huang, G. W., Zhang, Q., \& Xu, G. C. (2014). Real-time clock offset prediction with an improved model. GPS Solutions, 18(1), 95-104. https://doi. org/10.1007/s10291-013-0313-0.

Huang, G. W., Cui, B. B., Zhang, Q., et al. (2018). Real-time clock offset prediction model with periodic and neural network corrections. Journal of Astronautics, 39(01), 83-88. https://doi.org/10.3873/j.issn.1000-1328.2018.01.011.

Ma, Z. X., Yang, L., \& Jia, X. L. (2017). Research on prediction and characterization of periodic variations in BDS satellite clocks. Journal of Geodesy and Geodynamics, 37(03), 292-296. https://doi.org/10.14075/j.jgg.2017.03.015.

Sun, D. S., Lu, Z. P., Wang, Y. P., et al. (2016). A method of satellite clock bias prediction considering periodic errors and stochastic characteristics. Journal of Geodesy and Geodynamics, 36(12), 1078-1082. https://doi.org/10.14075 /j.jgg.2016.12.010

Wang, B. (2016). Analysis of BDS satellite clock in orbit, modeling and its prediction research (p. 2016). Wuhan: Wuhan University.

Wang, Y. P. (2017). Research on modeling and prediction of the satellite clock bias and performan evaluation of GNSS satellite clocks. Zhengzhou: PLA Information Engineering University.

Wang, Y. P., Lu, Z. P., Sun, D. S., et al. (2016). A new navigation satellite clock bias prediction method based on modified clock-bias quadratic polynomial model. Acta Astronomica Sinica, 57(01), 78-90. https://doi.org/10.15940/j.c nki.0001-5245.2016.01.008.

Wang, Y. P., LV, Z. P., \& Wang, N. (2017). The Long-term Performance Analysis for On-board Atomic Clocks of BDS[J]. Acta Geodaetica et Cartographica Sinica, 46(02), 157-169. https://doi.org/10.11947/j.AGCS.2017.20160369.

Xiao, S. H., Wang, G. C., \&Tu, Y. (2006). The SSA + FBPF method and its application on extracting the periodic term from BeiDou satellite clock bais. Acta Geodaetica et Cartographica Sinica, 45(S2), 172-178. https://doi. org/10.11947/j.AGCS.2016.F039.

Zhang, S. L. (2003). The theoretical and application research on nonlinear semiparametric model. Wuhan: Wuhan University.

Zhang, B., Ou, J. K., Yuan, Y. B., et al. (2007). Fitting method for GPS satellite clock errors using wavelet and spectrum analysis. Surveying and Information Science of Wuhan University, 08, 715-718. https://doi.org/10.3969/j. issn.1671-8860.2007.08.015.

Zhang, J., Zhou, W., Xuan, Z. Q., et al. (2013). Selection of periodic items and its performance in the forcasting model of satellite clock bias. Acta Astronomica Sinica, 54(03), 282-290. https://doi.org/10.3969/j. issn.0001-5245.2013.03.008

Zheng, Z. Y., Dang, Y. M., Lu, X. S., et al. (2010). Prediction model with periodic item and its application to the prediction of gps satellite clock bias. Acta Astronomica Sinica, 51(01), 95-102. https://doi.org/10.15940/j.c nki.0001-5245.2010.01.012.

Zhou, P. Y., Du, L., Lu, Y., et al. (2015). Periodic variations of BeiDou satellite clock offsets derived from multi-satellite orbit determination. Acta Geodaetica et Cartographica Sinica, 44(12), 1299-1306. https://doi.org/10.11947 /j.AGCS.2015.20150183.

Zhou, P. Y., Du, L., Fang, S. C., et al. (2016). Analysis of characteristics of QZSS satellite orbit and clock products during yaw attitude model switching. Acta Geodaetica et Cartographica Sinica, 45(03), 274-281. https://doi. org/10.11947/j.AGCS.2016.20150386.

Zhu, X. W., Xiao, H., Yan, S. W., et al. (2008). The Kalman algorithm used for satellite clock offset prediction and its performance analysis. J Astronaut, 29(03), 966-970. https://doi.org/10.3873/j.issn.1000-1328.2008.03.045.

\section{Publisher's Note}

Springer Nature remains neutral with regard to jurisdictional claims in published maps and institutional affiliations.

\section{Submit your manuscript to a SpringerOpen ${ }^{\circ}$ journal and benefit from:}

- Convenient online submission

- Rigorous peer review

- Open access: articles freely available online

- High visibility within the field

Retaining the copyright to your article

Submit your next manuscript at springeropen.com 